\title{
LA COLLABORATION INTERPROFESSIONNELLE COMME MODALITÉ DE RÉSOLUTION DES IMPASSES THÉRAPEUTIQUES EN PÉDOPSYCHIATRIE : RECHERCHE-ACTION PARTICIPATIVE INTERPROFESSIONAL COLLABORATION AS A MODALITY TO RESOLVE THERAPEUTIC IMPASSES IN PEDOPSYCHIATRY: A PARTICIPATORY RESEARCH-ACTION
}

\author{
Lyne Bordeleau et Jeannette LeBlanc
}

Volume 40, numéro 2, 2019

URI : https://id.erudit.org/iderudit/1065912ar

DOI : https://doi.org/10.7202/1065912ar

Aller au sommaire du numéro

Éditeur(s)

Revue québécoise de psychologie

ISSN

2560-6530 (numérique)

Découvrir la revue

Citer cet article

Bordeleau, L. \& LeBlanc, J. (2019). LA COLLABORATION

INTERPROFESSIONNELLE COMME MODALITÉ DE RÉSOLUTION DES IMPASSES

THÉRAPEUTIQUES EN PÉDOPSYCHIATRIE : RECHERCHE-ACTION

PARTICIPATIVE. Revue québécoise de psychologie, 40(2), 263-284.

https://doi.org/10.7202/1065912ar

\section{Résumé de l'article}

Cet article présente les fondements, la méthode et les résultats d'une recherche-action participative visant à comprendre comment des professionnels travaillant dans une clinique de pédopsychiatrie construisent leur pratique de collaboration interprofessionnelle, le contexte dans lequel se construit cette pratique et comment ils peuvent améliorer la résolution des impasses thérapeutiques en réfléchissant sur cette pratique. Un traitement qualitatif, à partir du procédé d'analyse thématique de données recueillies au cours de périodes de réflexion en équipe et d'une entrevue de groupe, a permis d'identifier sept déterminants du processus de collaboration

interprofessionnelle regroupés selon trois thèmes : 1) l'engagement, 2) les interactions et 3) le contexte de pratique. Six déterminants favorisent le travail de collaboration interprofessionnelle, alors qu'un y fait obstacle. La mise en évidence des déterminants au cours du déroulement de la recherche a un impact positif sur le processus de collaboration interprofessionnelle et sur la résolution des impasses thérapeutiques. Les forces et les limites de la recherche, ses retombées pour la pratique et des pistes pour de futures recherches sont présentées. 


\section{LA COLLABORATION INTERPROFESSIONNELLE COMME MODALITÉ DE RÉSOLUTION DES IMPASSES THÉRAPEUTIQUES EN PÉDOPSYCHIATRIE : RECHERCHE-ACTION PARTICIPATIVE}

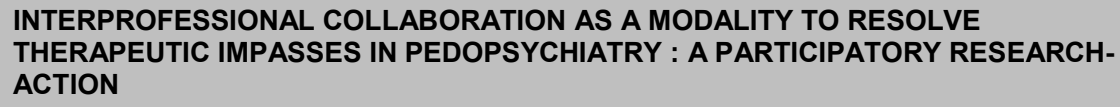

Lyne Bordeleau

Université de Sherbrooke

Jeannette LeBlanc ${ }^{1}$

Université de Sherbrooke

Malgré la promotion faite en faveur du bien-être global de la population mondiale, les statistiques rapportées par l'Organisation mondiale de la santé (2014) montrent que la maladie mentale demeure une des premières causes d'invalidité à travers le monde et se manifeste, malheureusement, assez rapidement lors du développement des enfants et adolescents. En effet, la moitié de toutes les maladies mentales apparaissent avant l'âge de 14 ans (Ministère de la Santé et des Services sociaux [MSSS], 2015). Au Canada, on estime à $14 \%$ à $25 \%$ le nombre d'enfants et d'adolescents touchés par la maladie mentale (Manion, 2010).

En 2012, le rapport de Fleury et Grenier sur l'état de situation en santé mentale au Québec insiste sur l'importance d'intervenir tôt et intensivement auprès des jeunes présentant un trouble mental modéré ou grave. Les auteurs justifient cette prise de position par le fait que des recherches longitudinales démontrent que les troubles mentaux réduisent les champs d'activité de ces jeunes ainsi que leur capacité d'agir, ont des impacts importants sur leur vie familiale et persistent à l'âge adulte lorsqu'ils ne sont pas traités, entraînant des coûts sociaux, psychologiques et financiers importants. Depuis une dizaine d'années, des mesures ont été prises au Québec afin d'améliorer l'accès et la continuité des services pour les jeunes aux prises avec des troubles mentaux. Le Plan d'action en santé mentale (PASM) 2005-2010 (MSSS, 2005) a préconisé des dispositions visant à développer les services de première ligne et à introduire des pratiques de soins axées sur la collaboration. En renforçant les services offerts par les partenaires intervenants en première ligne (milieu scolaire, centre local de services communautaires [CLSC], centre de la petite enfance [CPE], clinique médicale), les cliniques de pédopsychiatrie et les cliniques spécialisées deviennent disponibles pour offrir des traitements aux jeunes présentant des troubles mentaux plus sévères. Le travail axé sur la collaboration interprofessionnelle dans les équipes de soins et entre les divers partenaires vise à offrir des soins

1. Adresse de correspondance: Département de psychologie, Université de Sherbrooke, 2500, boulevard Université, Sherbrooke (QC), J1K 2R1. Courriel : Jeannette.leblanc@usherbrooke.ca 
accessibles, continus et complets. Ces mêmes orientations sont maintenues par le PASM 2015-2020 (MSSS, 2015).

En 2010, dans son rapport d'évaluation, le MSSS rapporte des retombées positives découlant de l'implantation des mesures du PASM pour la clientèle jeunesse. Cependant, certains obstacles concernant les pratiques de collaboration entre les professionnels et entre les institutions demeurent. Les défis associés à la mise en place de pratiques de collaboration ne sont pas exclusifs au Québec. Une revue de la littérature réalisée par Fuller et al. (2011), regroupant 119 études publiées entre 1998 et 2009 dans six régions du monde (Australie, Nouvelle-Zélande, Royaume-Uni, Europe, États-Unis et Canada) auprès d'une clientèle en santé mentale, indique un lien positif entre la collaboration interprofessionnelle et trois éléments associés aux soins, soit une réduction significative des symptômes cliniques, une plus grande efficacité des services et une diminution des coûts des services. Cependant, malgré la reconnaissance de l'efficacité de la collaboration interprofessionnelle, l'implantation de cette pratique dans le milieu de la santé demeure difficile (Murray, Silver, Patel, Dupuis, Hayes et Davis, 2008; Sicotte, D'Amour et Moreault, 2002; Suter, Arndt, Arthur, Parboosingh, Taylor et Deutschlander, 2009).

Cet article porte sur les services offerts pour traiter les troubles mentaux plus sévères chez les jeunes. II s'intéresse plus spécifiquement à la collaboration interprofessionnelle comme moyen permettant d'augmenter la qualité de ces services en contribuant à la résolution des impasses thérapeutiques vécues par des professionnels travaillant en clinique de pédopsychiatrie (voir Bordeleau et LeBlanc, 2017, pour une revue de littérature). Nous présentons d'abord les sources possibles d'impasses thérapeutiques en clinique de pédopsychiatrie et introduisons le concept de la collaboration interprofessionnelle. Nous faisons ensuite état d'une recherche-action participative réalisée en clinique de pédopsychiatrie qui visait à mieux comprendre comment des professionnels peuvent, en s'engageant dans une attitude réflexive sur leur processus de collaboration interprofessionnelle, résoudre les impasses thérapeutiques rencontrées dans leur pratique. Une présentation des forces et limites de la recherche, les retombées pour la pratique et des pistes pour de futures recherches closent l'article.

\section{LES IMPASSES THÉRAPEUTIQUES ET LA COLLABORATION INTERPROFESSIONNELLE}

Au Québec, les services spécialisés de deuxième ligne en santé mentale pour les jeunes sont offerts par les professionnels travaillant en clinique de pédopsychiatrie. Ces services s'adressent aux jeunes âgés de 
0 à 17 ans présentant des problèmes de santé mentale modérés à sévères qui ne peuvent pas être traités par les partenaires de la première ligne. Les cliniques regroupent des professionnels de plusieurs disciplines: éducation spécialisée, ergothérapie, neuropsychologie, orthophonie, orthopédagogie, psychoéducation, psychologie, psychiatrie, soins infirmiers, travail social, thérapie conjugale et familiale. L'organisation des services et la constitution des équipes varient d'une clinique à une autre. Par leur mandat et leur constitution, ces cliniques offrent un contexte pertinent pour l'exploration des impasses thérapeutiques et de la collaboration interprofessionnelle (Lesinskiene, Senina et Ranceva, 2007).

En effet, dans l'intervention auprès des enfants et des adolescents, les professionnels risquent beaucoup de vivre des sentiments et des réactions pouvant influencer leur jugement clinique et leur comportement de façon potentiellement contre-thérapeutique (Rasic, 2010). Ils doivent en effet tenir compte de collègues et de partenaires aux cultures, règles et rythmes de fonctionnement différents, ce qui peut provoquer des réactions ayant des conséquences sur le travail de collaboration et l'orientation de l'intervention (Nadeau et al., 2012). Les difficultés de collaboration peuvent générer, à leur tour, des effets sur la continuité des services offerts aux jeunes et nuire à la prise en charge des professionnels et des partenaires qui gravitent autour de cette clientèle (Rousseau, Nadeau, Pontbriand, Johnson-Lafleur, Measham et Broadhurst, 2014). L'intervention en clinique de pédopsychiatrie présente aussi des défis particuliers associés aux caractéristiques des jeunes: la difficulté d'établir le diagnostic d'une personne en développement, la présence d'une comorbidité psychiatrique chez plus de $50 \%$ des jeunes, le taux élevé d'abandon des traitements et l'engagement indispensable des parents pour que le jeune puisse recevoir les soins (Fleury et Grenier, 2012). De plus, les transformations entreprises dans le réseau de la santé et des services sociaux québécois depuis les vingt dernières années, malgré certains effets positifs, ont contribué à l'alourdissement de la clientèle traitée en clinique de pédopsychiatrie (MSSS, 2010). Tous ces éléments sont des sources possibles d'impasses thérapeutiques pour les professionnels travaillant dans ces cliniques. L'impasse thérapeutique est définie ici comme une conversation thérapeutique entre un professionnel et son patient dans laquelle le thérapeute n'arrive plus à choisir, parmi ses interventions habituelles, celles qui pourraient faire évoluer le processus (Rober, 1999). Dans le cadre de cette recherche, les impasses thérapeutiques s'observent lors des réunions d'équipe, lorsque les professionnels manifestent des difficultés à collaborer et que ces difficultés de collaboration interprofessionnelle ne permettent plus aux membres de l'équipe de choisir les interventions qui permettraient de faire évoluer le patient. La collaboration interprofessionnelle dans les équipes de soins de santé est, pour sa part, définie comme le processus par lequel des 
professionnels interdépendants structurent une action collective afin de répondre aux besoins des patients (D’Amour, 1997).

Enfin, le modèle de D’Amour, Goulet, Labadie, San Martín-Rodriguez et Pineault (2008) constitue le cadre conceptuel de cette recherche. Ce modèle s'organise autour de quatre dimensions générales interreliées auxquelles sont associés dix déterminants de la collaboration interprofessionnelle. Ces déterminants se déclinent ainsi: objectif commun, orientation centrée sur le client par rapport aux autres allégeances, centralité, leadership, soutien pour l'innovation, connectivité, connaissance et confiance mutuelle, outils de formalisation et échanges d'informations.

\section{OBJECTIFS}

La recherche vise à comprendre comment des professionnels travaillant dans une clinique de pédopsychiatrie construisent leur pratique de collaboration interprofessionnelle, le contexte dans lequel se construit cette pratique et comment ils améliorent la résolution des impasses thérapeutiques rencontrées en s'engageant dans une activité de réflexion sur cette pratique. La recherche répond aux trois questions suivantes : 1) Quels sont les déterminants du processus de collaboration interprofessionnelle pouvant être identifiés lors de discussions de cas sujettes à une impasse thérapeutique? 2) Quels sont les déterminants qui favorisent ou qui font obstacle à la collaboration interprofessionnelle lors d'une discussion impliquant une impasse thérapeutique? Et 3) quel impact a la mise en évidence de ces déterminants sur la résolution des impasses thérapeutiques pendant le déroulement de la recherche?

\section{DÉMARCHE MÉTHODOLOGIQUE}

La recherche-action participative (RAP) est la démarche choisie pour comprendre la pratique et le contexte de pratique de collaboration interprofessionnelle de professionnels d'une clinique de pédopsychiatrie, et tenter d'en améliorer le fonctionnement lorsqu'ils examinent les impasses thérapeutiques rencontrées. La RAP permet de recueillir et d'analyser systématiquement des données afin de générer des connaissances qui suscitent des changements dans la pratique (MacDonald, 2012). Selon Fradet (2013), il s'agit d'un choix pertinent pour l'étude de situations vécues dans le milieu de la santé et des services sociaux. En effet, cette méthode permet au chercheur de s'associer à une communauté, d'assurer son intérêt pour les questions de recherche, la collecte, l'analyse et l'interprétation des données, des conditions permettant aux membres de la communauté d'accroître leur capacité d'identifier et de résoudre leurs problèmes, et permet aux gestionnaires de mobiliser leurs ressources et 
d'améliorer leurs politiques afin d'influencer les pratiques des professionnels (Jagosh et al., 2012). La RAP se réalise à partir de cycles d'actions et de cycles de réflexions (Baum, MacDougall et Smith, 2006).

La rigueur méthodologique est assurée par la mise en place des stratégies suivantes: constante veille afin que chaque participant puisse s'exprimer et valider de manière continue sa compréhension des propos retenus; implication constante des deux auteurs dans le processus d'analyse; validation de l'analyse des données avec l'ensemble des professionnels à la fin du processus de collecte de données; utilisation de plusieurs sources de collecte de données pour assurer la triangulation (Lincoln et Guba, 1985). Enfin, la recherche respecte le code des comités d'éthique et de recherche du Centre hospitalier et celui de l'Université de Sherbrooke.

\section{RECRUTEMENT ET PARTICIPANTS}

Pour mener cette recherche, nous nous sommes associés à une équipe de professionnels en pédopsychiatrie de la région métropolitaine de Montréal $^{2}$ ayant accepté d'examiner sa pratique interprofessionnelle et de s'engager dans une attitude réflexive sur les stratégies utilisées lors des situations d'impasses thérapeutiques (Baum et al., 2006). Un premier contact a été fait auprès du responsable des services en santé mentale et psychiatrie jeunesse et du responsable des pratiques professionnelles de la clinique. Le projet de recherche a ensuite été présenté aux deux pédopsychiatres de la clinique. Après avoir obtenu l'aval des pédopsychiatres, le projet a été exposé aux membres de l'équipe afin de valider leur engagement à participer à la démarche. Cette équipe de professionnels a été choisie parce qu'elle manifestait de l'intérêt à comprendre son processus de collaboration interprofessionnelle lors des situations d'impasses thérapeutiques et désirait jouer un rôle actif dans la recherche (Meyer, 2000). Le travail de terrain a été fait par le premier auteur de cet article, qui a joué un rôle de catalyseur plutôt qu'un rôle d'expert (Cassell et Johnson, 2006).

L'équipe sélectionnée se compose de deux pédopsychiatres, d'un psychologue, d'un orthophoniste, d'un travailleur social, d'un orthopédagogue, d'un psychoéducateur, de trois infirmiers et de quatre éducateurs spécialisés. La clinique est en activité du lundi au vendredi, et les intervenants se relaient $24 \mathrm{~h}$ sur 24 . L'expérience professionnelle et le nombre d'années cumulées dans cette clinique par chacun des professionnels sont variables de l'un à l'autre. Le nombre de participants à chacun des cycles d'actions et des cycles de réflexions varie de 6 à 11

2. Les auteurs de l'article remercient l'équipe participante, sans laquelle cette recherche n'aurait pu être réalisée. 
professionnels en raison des congés (maladie, maternité) et des problématiques discutées. Deux professionnels ont participé à tous les cycles d'actions et de réflexions, quatre professionnels ont été présents à cinq des six cycles et six professionnels n'ont assisté qu'à deux des cycles. L'ensemble du personnel de la clinique a participé à l'entrevue de groupe qui a lieu à la fin du processus.

\section{COLLECTE DE DONNÉES}

Quatre types de données sont recueillies : l'enregistrement sur bande vidéo des 6 réunions d'équipe d'une durée de 75 minutes; l'enregistrement audio de 6 périodes de réflexion en équipe d'une durée variant de 30 à 60 minutes, selon la disponibilité des professionnels; l'enregistrement audio de l'entrevue de groupe d'une durée de 75 minutes et les notes descriptives et théoriques de la chercheuse.

La collecte de données s'est déroulée en deux étapes : 1) Six cycles d'actions et de réflexions sont mis en place au rythme d'une fois par mois pendant une période de neuf mois incluant une pause pour la saison estivale. Chaque cycle d'actions et de réflexions est constitué d'abord par la participation à la réunion d'équipe, suivie, le lendemain, par la participation à une rencontre au cours de laquelle sont visionnées des séquences de la réunion d'équipe sélectionnées préalablement par le premier auteur de l'article. 2) Une entrevue de groupe a lieu deux mois après le dernier cycle d'actions et de réflexions.

Les séquences visionnées sont choisies à partir de la technique de l'incident critique (TIC) et d'une grille d'observation inspirée des déterminants de la collaboration interprofessionnelle du modèle de D'Amour et al. (2008). L'incident critique est une activité humaine observable qui se produit dans une situation où l'intention de l'acte et ses conséquences sont suffisamment précises pour laisser peu de doutes aux observateurs (Flanagan, 1954). La TIC permet de recueillir des informations riches, concrètes et détaillées sur l'activité étudiée, en l'occurrence la pratique de collaboration interprofessionnelle lors de la gestion des impasses thérapeutiques en réunion (Walker, 2015), et de l'analyser rigoureusement. La TIC fournit aussi une structure à la démarche de recherche-action (Chou, Kwee, Buchanan et Lees, 2016). Les séquences varient de deux à six minutes. Elles servent de point de départ aux périodes de réflexion en équipe. Les professionnels visionnent ensemble une séquence à la fois, puis s'engagent dans une attitude réflexive sur leur fonctionnement d'équipe pendant cette séquence, leur efficacité dans la résolution de l'impasse thérapeutique et l'identification des stratégies visant à améliorer leur fonctionnement. 
Des notes descriptives concernant le déroulement des rencontres (personnes présentes, interactions, climat) et des notes théoriques (interrogations, hypothèses, intuitions) sont consignées par la chercheuse.

\section{ANALYSE DES DONNÉES}

Les données sont soumises à une analyse thématique conformément à la méthode de Paillé et Mucchielli (2008). La première étape consiste à extraire des données le contenu ayant un lien avec les objectifs de la recherche. Ces données sont ensuite codées, puis ces codes sont découpés, analysés et regroupés sous des thèmes homogènes. Dans une deuxième étape, les thèmes sont mis en lien les uns avec les autres et explorés à l'aide du modèle théorique du processus de collaboration interprofessionnelle de D'Amour et al. (2008). La troisième étape consiste à valider les résultats ainsi obtenus auprès des participants lors de l'entrevue de groupe. Finalement, la quatrième étape nous a permis, en relevant les récurrences, les divergences, les oppositions et les regroupements dans l'ensemble des données recueillies (séquences extraites des réunions d'équipe, verbatim des périodes de réflexion, entrevue de groupe et notes de la chercheuse), de définir trois thèmes majeurs qui reviennent régulièrement dans le discours des participants. Sept déterminants de la collaboration interprofessionnelle ont également pu être inférés de ces thèmes.

\section{RÉSULTATS}

Trois thèmes sont retenus pour décrire la pratique de collaboration interprofessionnelle de l'équipe participante lors de leurs discussions de cas impliquant une impasse thérapeutique : l'engagement, les interactions et le contexte de pratique. Sept déterminants sont inférés de ces thèmes. Cette section expose les thèmes et les déterminants favorisants ou faisant obstacle à la collaboration interprofessionnelle. Les déterminants sont illustrés à l'aide des séquences sélectionnées (incidents critiques) pendant les réunions d'équipe. La mise en évidence des déterminants sur le travail de l'équipe est ensuite abordée.

Thèmes et déterminants du processus de collaboration interprofessionnelle

\section{L'engagement}

L'engagement, tel que décrit par les participants, correspond à la façon dont les professionnels s'engagent dans la discussion et se manifeste par les comportements suivants: prendre la parole pour transmettre des informations concernant la situation discutée, partager une réflexion quant au choix d'une intervention et son impact, proposer une piste d'intervention 
qui cherche à rallier les professionnels, inviter au développement d'idées en posant des questions et écouter l'opinion des autres même lorsqu'elle diffère sensiblement de la leur. Un déterminant est déduit du thème de l'engagement dans le cadre de cette recherche et y est favorable.

Les résultats montrent que le déterminant objectif de la discussion, partagé et orienté vers les besoins du patient, permet aux professionnels de s'engager dans la discussion en prenant la parole à tour de rôle et en s'écoutant mutuellement. Leurs ressources sont alors mises à la disposition de l'équipe pour chercher à résoudre l'impasse thérapeutique rencontrée. Ce déterminant s'observe lors d'une discussion concernant un enfant récemment hospitalisé. L'objectif de la discussion, qui est de comprendre la nature des difficultés de l'enfant, est partagé par tous les professionnels présents et orienté vers les besoins de l'enfant. L'impasse thérapeutique est suscitée par la perception qu'ont les professionnels du diagnostic de l'enfant. Certains considèrent qu'il y a un trouble neurodéveloppemental, alors que d'autres considèrent avoir affaire à des difficultés d'ordre affectif.

Faisant le lien avec ce qui a été dit précédemment par Thomas ${ }^{3}$ au sujet du diagnostic de l'enfant ${ }^{4}$, Émile rapporte des observations qui ne corroborent pas l'hypothèse de Thomas. Avant d'intervenir, Laurent encourage Émile à compléter sa lecture de la situation. Thomas reprend alors les observations d'Émile à partir d'arguments qui viennent appuyer sa propre hypothèse diagnostique. Sandrine et Ève partagent alors leurs observations, qui viennent appuyer à la fois certains éléments de l'hypothèse diagnostique de Thomas et celle d'Émile. Ensuite, Thomas s'interroge ouvertement sur la possibilité que l'équipe puisse intervenir auprès de l'enfant afin de vérifier son hypothèse diagnostique. Laurent reprend la suggestion de Thomas et propose un mode d'intervention qui permettrait de vérifier l'hypothèse de Thomas. II vérifie ensuite directement si Émile adhère à cette proposition et est d'accord à participer aux actions visant à vérifier l'hypothèse. Émile et les autres professionnels manifestent verbalement et non verbalement leur accord à la proposition.

Dans cette séquence, en tenant compte de leurs différences d'opinions, les professionnels prennent la parole pour donner leur opinion, inviter au développement d'idée, appuyer celle d'un autre ou encore suggérer une direction à l'intervention. Ils demeurent aussi à l'écoute de l'opinion des autres même si elle diffère de la leur.

3. Les noms des participants ont été remplacés par des noms fictifs afin de préserver leur anonymat.

4. Le recours au genre masculin pour désigner tous les patients vise à empêcher leur identification. 


\section{Les interactions}

Le thème des interactions, tel que décrit par les participants, réfère à la manière dont les professionnels témoignent de leur interdépendance. Ceci s'observe lorsqu'un professionnel signale l'impact d'un comportement d'un autre collègue sur la participation des autres professionnels, souligne l'aide qu'un membre de l'équipe lui apporte ou l'influence qu'il a sur sa réflexion, valorise une proposition faite par un autre, encourage un collègue qui hésite à donner son opinion et se préoccupe de l'impact de ses paroles sur le bien-être des autres.

Deux déterminants sont inférés de ce thème : la connaissance que les professionnels ont les uns des autres et la confiance qu'ils se manifestent réciproquement. La connaissance que les professionnels ont les uns des autres réfère à la connaissance qu'ils ont de l'approche de soins de leurs collègues, des valeurs qu'ils soutiennent et de ce qu'ils peuvent attendre les uns des autres lors des moments de crise et d'impasse thérapeutique. La confiance réciproque touche à la confiance dans les compétences de leurs collègues quant à leur capacité d'assumer leurs responsabilités professionnelles. La présence de ces deux déterminants permet aux professionnels de se sentir en sécurité pour parler de façon constructive de l'impasse thérapeutique rencontrée et de tenir compte de leur interdépendance dans la recherche des solutions. L'importance de ces déterminants s'observe lors d'une discussion concernant un enfant qui présente une belle évolution sur le plan clinique, alors que la mère anticipe avec beaucoup d'anxiété le moment où elle reprendra l'enfant en charge. L'impasse thérapeutique est suscitée par des prises de position différentes sur la suite des interventions à réaliser. Certains pensent que l'enfant doit demeurer hospitalisé, tandis que d'autres pensent que le maintien de l'hospitalisation nuira à son évolution.

Judy, qui hésite à réintégrer l'enfant dans son milieu de vie, se dit préoccupée par les réactions de la mère à propos de la décision de mettre fin à l'hospitalisation de l'enfant. Laurent, sur un ton conciliant, dit partager la perception de Judy, mais croit que l'équipe aura le temps de travailler la difficulté avec la mère. Prenant le même ton conciliant, Sandrine se dit en accord avec la perception de Laurent. Judy sourit; elle semble rassurée par les propos de Laurent et Sandrine. Sandrine s'adresse ensuite à Ėve pour lui signifier que l'argument qu'elle a apporté plus tôt a beaucoup contribué à la rassurer quant à la décision de mettre fin à l'hospitalisation. Ėve acquiesce par un signe de tête qui laisse supposer qu'elle apprécie le commentaire de Sandrine. Laurent se tourne ensuite vers Félix qui a fait, plus tôt, une suggestion d'activités visant à aider l'enfant à visualiser la fin de son hospitalisation. II dit trouver l'idée intéressante et pertinente dans la situation. Sandrine s'interroge sur l'impact que pourrait avoir l'anxiété de la mère sur la réussite du retour de l'enfant dans son milieu. Laurent se montre intéressé à la question de Sandrine, qui l'amène à revoir sa façon d'intervenir avec la mère. II réalise qu'il pourrait prendre soin de la mère en 
lui nommant clairement les préoccupations identifiées par l'équipe. II se retourne ensuite vers Rémi, qui l'écoute attentivement, et il lui dit qu'il reparlera aussi avec la mère du fait qu'elle lui a téléphoné pour lui parler de sa détresse. Enfin, Alexandre dit qu'il pourra lui aussi contribuer à l'intervention en abordant avec l'enfant la fin de son hospitalisation. Tous démontrent verbalement et non verbalement leur intérêt à participer à l'intervention.

Ici, compte tenu de ces différentes positions, les professionnels poursuivent leur échange tout en prenant soin de ce qui les relie les uns aux autres. Ils valorisent une proposition faite par un autre, soulignent l'influence d'un membre de l'équipe sur sa réflexion et encouragent un collègue à donner son opinion.

\section{Le contexte de pratique}

Le thème contexte de pratique se rapporte, selon les participants, au contexte dans lequel la collaboration interprofessionnelle est exercée. II s'observe à partir des dispositifs mis en place pour faciliter la communication entre les professionnels, favoriser le partage d'objectifs de travail communs, maintenir l'engagement des professionnels dans la discussion et entretenir un climat favorable aux interactions entre les membres.

Quatre déterminants sont inférés de ce thème, dont trois favorisent une pratique de collaboration interprofessionnelle, et un lui nuit. L'adhérence par les membres de l'équipe à un cadre théorique psychanalytique clairement identifié et partagé, la reconnaissance d'une zone spécifique d'expertise à chacun et l'instauration d'un leadership orienté vers la collaboration favorisent la mise en place d'un contexte qui facilite la collaboration. Lorsqu'ils utilisent un même référent théorique pour décrire une situation vécue, les professionnels se comprennent mieux lors de leurs échanges. La reconnaissance d'une zone d'expertise spécifique à chacun encourage la contribution unique de chaque professionnel dans un rôle actif pour résoudre l'impasse thérapeutique. Enfin, la prise de position des leaders de l'équipe pour une pratique axée vers la collaboration encourage la cohésion du groupe autant en ce qui a trait à la collaboration interprofessionnelle qu'à sa mobilisation vers la recherche de solutions. Ces déterminants s'observent lors d'une discussion portant sur un jeune hospitalisé pour des idées suicidaires. Les professionnels parlent d'une lettre que le père a laissée lors de son propre suicide et de la nécessité d'aborder le contenu de la lettre avec le jeune compte tenu du désir déjà manifesté par celui-ci d'en prendre connaissance. L'impasse thérapeutique est suscitée par la difficulté qu'éprouvent les professionnels à aborder le sujet avec le jeune, et ce, malgré la décision prise en équipe de mener cette intervention. 
Sandrine explique l'impasse thérapeutique dans laquelle se trouve l'équipe : malgré la décision prise d'accompagner le jeune dans la lecture de la lettre, les professionnels ont de la difficulté à la mettre en action et ils ont tendance à transmettre la tâche au quart de travail suivant. Rémi et Ėve disent qu'ils n'ont pas réussi à aborder le sujet avec le jeune parce qu'il le refuse. Judy se reporte alors au cadre théorique que l'équipe s'est antérieurement donné et dit avoir l'impression que ce qui se joue actuellement au sein de l'équipe ressemble à l'ambivalence que vit le jeune. Celui-ci veut, à certains moments, lire la lettre, mais s'y oppose lorsque l'occasion se présente, ce qui ressemble un peu à ce que font les membres de l'équipe. Elle dit croire que le jeune peut légitimement avoir peur du contenu de la lettre. Elle demande ensuite s'ils ont lu la lettre. Rémi et Félix disent l'avoir lue et craindre que la lettre vienne envenimer la relation mère-fils, compte tenu de son caractère accusateur envers la mère. Laurent reconnaît que l'implication de Rémi et Félix auprès de l'enfant est plus grande que la sienne et que cela donne du poids à leurs craintes. II revient ensuite au cadre théorique en soulignant qu'il croit que la lettre pourrait effectivement éveiller l'agressivité entre la mère et le fils, mais que sa lecture pourrait aussi permettre de la traiter avant son retour à la maison. Félix met en doute la capacité de la mère d'être à l'écoute des besoins de son fils, bien qu'il soit capable de s'exprimer adéquatement. Alexandre, pour sa part, s'inquiète des capacités du jeune à reconnaître les émotions suscitées par la lecture de la lettre. Le jeune lui a dit qu'il connaissait le contenu de la lettre et qu'il ne voulait pas la lire devant un professionnel qui chercherait ensuite à l'analyser. En se référant au cadre théorique, Laurent dit avoir l'impression que le jeune se comporte avec l'équipe comme il le fait avec sa mère, en prenant un rôle d'autorité. II propose alors à l'équipe d'aborder la situation avec le jeune en essayant de se placer dans leur rôle d'adulte ayant les capacités de se donner les conditions nécessaires pour la lecture de la lettre. II rappelle qu'il est important de reconnaître que le jeune appréhende la lecture de la lettre et qu'ils seront présents pour l'aider à traverser cette épreuve, même si la situation est difficile pour eux. Les membres de l'équipe redonnent leur accord à la proposition.

Au cours de cette séquence, le cadre théorique sur lequel s'appuient les professionnels dans leurs interventions est ramené à l'avant-plan pour expliquer la situation vécue par les membres de l'équipe. II permet l'utilisation d'un langage commun et favorise le partage d'objectifs de travail commun. La reconnaissance de leur zone d'expertise spécifique les encourage à s'exprimer sur l'expérience personnelle que chacun vit avec l'enfant. Les leaders de l'équipe (Judy et Laurent) interviennent en encourageant la cohésion du groupe autour d'une recherche de solution partagée.

Le déterminant inféré du thème contexte de pratique qui nuit ici à la collaboration interprofessionnelle est lié aux difficultés d'animation de la réunion d'équipe. Lorsque l'animation ne permet pas aux membres de nommer et de réguler les réactions émotives suscitées lors des 
interactions avec les autres, les réactions retenues les empêchent d'être complètement disponibles pour participer et s'engager librement. Elles viennent alors teinter les interactions, rendant difficile la résolution de l'impasse thérapeutique. Ce déterminant s'observe lors d'une discussion concernant une impasse thérapeutique suscitée par des sentiments d'impuissance éveillés chez des professionnels par les comportements d'un enfant. Exceptionnellement, tous les professionnels de la clinique ont été convoqués à la réunion. L'enfant dont il est question a de grandes difficultés à être en relation avec les autres enfants de l'unité et avec les professionnels. II alterne entre des comportements agressifs (insulte, repousse, frappe, etc.) et de détresse (s'isole, pleure, etc.). Un professionnel a été blessé la veille de la réunion. L'impasse thérapeutique prend son origine d'un différend dans la perception des professionnels. Certains croient que les difficultés de l'enfant sont d'une telle ampleur qu'il est difficile, voire impossible de faire des interventions qui favoriseront son évolution clinique. D'autres pensent, au contraire, qu'il est encore possible de mener des interventions qui aideraient l'enfant à développer une meilleure gestion de ses émotions, suggérant qu'ils peuvent encore en faire plus pour l'aider.

\begin{abstract}
S'adressant aux autres membres de l'équipe, Thomas se demande s'il n'y a pas lieu de modifier les stratégies d'intervention utilisées avec l'enfant. II croit que les professionnels devraient essayer d'intervenir avant que les comportements deviennent trop agressifs. Les visages de Julien, Félix et Ève demeurent impassibles devant la proposition. Thomas termine son interprétation de la situation sans tenir compte de leur absence de réaction. Judy se retourne ensuite vers les autres professionnels et leur demande s'ils sont d'accord avec la suggestion de Thomas. Félix acquiesce timidement, mais rapidement, Julien, Ėve et lui évoquent le manque de collaboration de l'enfant. Rémi déclare qu'il est en accord avec l'idée de Thomas d'essayer d'intervenir auprès de l'enfant avant le déclenchement de sa crise, mais il ajoute que ce n'est pas toujours possible, compte tenu de la vitesse avec laquelle arrivent les gestes agressifs. Personne ne réagit à l'intervention de Rémi. Judy propose alors qu'une entente soit faite avec l'enfant pour convenir d'une façon de faire lorsqu'on s'aperçoit que la crise approche. Puis, elle demande aux professionnels si cette stratégie a déjà été essayée. Julien, Félix et Ėve donnent des exemples démontrant qu'ils l'ont déjà essayée et qu'elle n'a pas fonctionné. Certains professionnels rient sans que l'on comprenne pourquoi. Laurent donne une explication théorique du comportement de l'enfant, insistant sur l'importance d'ajuster l'intervention en fonction des besoins de l'enfant. Les visages de Julien, Ėve et Félix restent fermés. Quatre professionnels gardent le silence pendant la durée de l'échange.
\end{abstract}

Dans cette séquence, les comportements non verbaux des professionnels témoignent d'un inconfort important pendant la discussion. Judy, qui est l'initiatrice de cette réunion, démontre des difficultés à l'animer et personne d'autre ne prend la relève. Plusieurs semblent 
demeurer dans un état de dérégulation émotionnelle qui rend difficile leur engagement dans la discussion et ne permet pas la résolution de l'impasse.

Impact de la recherche sur le travail de l'équipe

Trois impacts de la recherche-action sont relevés par les professionnels au moment de la rencontre de groupe ayant eu lieu à la fin du processus: une prise de position pour la collaboration interprofessionnelle, une plus grande capacité de l'équipe à réfléchir sur son action et une meilleure organisation de son temps en réunion.

Prise de position pour la collaboration interprofessionnelle

L'aval rapidement obtenu des deux pédopsychiatres pour participer à une recherche-action impliquant des cycles d'actions et de réflexions suggère l'intérêt de ces leaders pour une pratique orientée vers la collaboration interprofessionnelle. II est possible de supposer que ces personnes, consciemment ou non, adhèrent à l'idée que la collaboration interprofessionnelle nécessite un processus complémentaire d'apprentissage pour se développer. Les membres de l'équipe ont aussi nommé leur intention de poursuivre leur démarche de réflexion sur leur fonctionnement d'équipe qu'ils avaient par ailleurs déjà débuté avant la mise en place de cette recherche-action participative.

\section{Réflexion sur l'action}

La capacité des professionnels de porter un regard sur l'action dans le cadre de la recherche a nécessité une période d'apprentissage. En les invitant rapidement à devenir observateurs de leurs actions lors de courtes séquences, la méthode de l'incident critique leur a permis de porter attention aux actions posées, d'évaluer ces actions et de chercher des interventions pouvant encore mieux répondre à leurs buts. Ils ont ainsi été invités à se centrer sur leurs interactions lors de séquences et à se poser les questions suivantes: "Est-ce que les interventions observées lors de l'échange nous permettent d'atteindre l'objectif? "; "Est-ce que les interactions observées entre les professionnels nous permettent d'avoir accès à l'expertise de chacun? "; " Est-ce que le déroulement des échanges contribue à entretenir des relations constructives entre les professionnels? ". Ils ont constaté rapidement que la démarche stimule une réflexion constructive quant à leur façon de travailler en équipe. La chercheuse, comme membre externe et neutre, les a aidés à demeurer centrés sur leur action et à apprendre de cette action. 


\section{Organisation du temps en réunion}

À travers la répétition des cycles d'actions et de réflexions, les professionnels ont l'occasion d'observer certaines difficultés sur le plan de l'animation des discussions menant à une impasse thérapeutique. Ils reviennent, à quelques reprises, sur des décisions prises lors des réunions d'équipe et sur leur mise en œuvre pour constater qu'ils ont rarement le temps de préciser l'orientation des prochaines interventions et les responsabilités de chacun. Lors du cinquième cycle d'actions et de réflexions, ils conviennent d'une stratégie (installation d'une affiche sur laquelle il est inscrit "Qui fait quoi ») comme rappel visuel pendant la réunion. Cette stratégie pallie partiellement les difficultés d'animation liées à l'organisation du temps. De telles stratégies de suppléance peuvent faciliter la gestion des échanges et répondre aux besoins des membres de " suivre une démarche cohérente et satisfaisante (p.119) » (St-Arnaud, 2008).

\section{DISCUSSION}

L'analyse a permis d'identifier six déterminants de la collaboration interprofessionnelle ayant une influence positive sur le processus de collaboration de cette équipe de pédopsychiatrie, soit la présence d'un objectif partagé orienté vers les besoins du patient, la connaissance mutuelle des professionnels, la confiance réciproque, l'adoption par les professionnels d'un cadre théorique partagé, la reconnaissance d'une zone spécifique d'expertise à chacun des membres de l'équipe et un leadership orienté vers la collaboration. Ces résultats vont dans le même sens que ceux obtenus par d'autres chercheurs. En effet, six experts en collaboration interprofessionnelle du Québec affirment que la collaboration interprofessionnelle est possible lorsque des professionnels interagissent de manière à élaborer une vision commune d'une situation problématique et un plan d'intervention orienté vers les besoins du patient (Careau, Vincent et Swaine, 2011). Hesjedal, Hetland et Iversen (2015) arrivent aux mêmes conclusions lors d'une étude qualitative réalisée en Norvège avec 13 professionnels, enseignants et travailleurs sociaux intervenant auprès d'enfants dont les difficultés nécessitent une intervention multidisciplinaire, à savoir que la présence d'objectifs communs orientés vers les besoins de l'enfant favorise le travail de collaboration interprofessionnelle. Sidani et Fox (2014) attestent aussi que les interventions visant la promotion des soins centrés sur le patient sont les plus susceptibles de rallier l'ensemble des professionnels d'une équipe. Andvig, Syse et Severinsson (2014) signalent l'importance pour les professionnels de se connaître mutuellement, tandis que McInnes, Peters, Bonney et Halcomb (2015) et Zwarenstein et Reeves (2006) mettent en évidence les conséquences positives de l'existence d'une confiance dans les compétences professionnelles des uns et des autres entre les professionnels. 
Le succès d'une équipe n'est toutefois pas seulement fonction des capacités des membres de l'équipe et des ressources disponibles; la réalisation avec succès de leurs tâches dépend aussi des processus d'interactions entre les membres (Marks, Mathieu et Zaccaro, 2001). Dans ce sens, l'importance des dispositifs favorisant la mise en place d'un contexte de pratique axée sur la collaboration interprofessionnelle est soulignée par d'autres chercheurs. En effet, dans une recherche réalisée auprès de 30 médecins résidents qui participaient à des simulations d'intervention en équipe dans une clinique d'urgence, Gardner, Scott et AbdelFattah (2016) indiquent que les équipes partageant, entre autres, un modèle théorique commun sont plus performantes. McComb et Simpson (2014) vont dans le même sens, affirmant que l'existence d'un modèle théorique offre une structure de connaissances aux équipes et les aide à mieux collaborer. Pour leur part, Sims, Hewitt et Harris (2015), qui s'intéressent aux processus sous-jacents au travail d'équipe interprofessionnelle, concluent que lorsque les rôles et les responsabilités des membres de l'équipe sont clairement identifiés, il devient plus facile pour les professionnels de différentes disciplines de collaborer les uns avec les autres, de coordonner leur travail de manière à réduire les duplications et les omissions des services et d'offrir une prestation plus efficace des soins. Enfin, dans le cadre d'une recherche-action réalisée dans un département de santé mentale en Australie, Beckett, Field, Molloy, Yu, Holmes et Pile (2013) montrent que le leadership qui encourage le développement d'équipes orientées vers des visions communes et des objectifs communs, guidé par des valeurs de respect et de collégialité, est associé à l'amélioration des performances des équipes ainsi qu'au bien-être de leurs membres.

L'analyse met aussi en évidence un déterminant faisant obstacle au processus de collaboration interprofessionnelle : les difficultés d'animation. Cet obstacle à la collaboration interprofessionnelle fait également écho aux résultats obtenus par d'autres auteurs (Careau et al., 2011; Washington, Guo, Albright, Lewis, Oliver et Demiris, 2017). Les procédés d'animation correspondent à un ensemble de comportements dont certains contribuent spécifiquement à éviter ou à gérer les situations qui font obstacle aux échanges entre les membres d'une équipe (St-Arnaud, 2008). En aidant à extérioriser les émotions et à objectiver les interventions, ces procédés (donner la parole, susciter ou réfréner la participation, sensibiliser au temps, reformuler, faire un résumé, expliciter, etc.) facilitent les interactions entre les membres, leur permettant de se recentrer sur leurs cibles de travail (St-Arnaud, 2008). De plus, il y a, selon Toegel et Barsoux (2016), de grands bénéfices à s'occuper rapidement des situations potentiellement conflictuelles : cela évite que le conflit prenne de l'ampleur, favorise une plus grande participation des membres, améliore la recherche de solutions et permet une meilleure prise de décision. 
Enfin, l'analyse montre que la mise en évidence des déterminants de collaboration interprofessionnelle pendant le déroulement de la recherche a eu des impacts sur la résolution des impasses thérapeutiques au sein de l'équipe. Dès le début, les professionnels reconnaissent vivre des impasses thérapeutiques dans leurs interventions et souhaitent améliorer leur façon de travailler ensemble afin d'élargir l'éventail de leurs stratégies et mieux gérer les impasses thérapeutiques rencontrées. II est généralement admis que l'impasse thérapeutique émerge à partir de deux sources principales: 1) les caractéristiques pathologiques du patient et leurs effets sur le déroulement de la thérapie et l'établissement de l'alliance thérapeutique, et 2) la manière dont le thérapeute y réagit (Bessette, 2010). Cette recherche-action a ramené à l'avant-plan l'existence d'une troisième source de l'impasse thérapeutique, soit celle qui prend racine au sein même du travail en équipe. Les tâches d'évaluation et de traitement en clinique de pédopsychiatrie impliquent des interactions d'interdépendance et d'interinfluence entre des professionnels ayant des expertises différentes (Fleury et Grenier, 2012). Les impasses thérapeutiques apparaissent dans le cadre de cette recherche lorsque les professionnels doivent poser un diagnostic multidisciplinaire et qu'ils n'arrivent plus à faire consensus. Elles émergent aussi lorsque plusieurs d'entre eux sont impliqués dans l'intervention auprès du patient et qu'ils ne parviennent pas à adopter une orientation commune quant aux services à offrir. Ces observations rejoignent celles relevées par Molin, Graneheim, Ringner et Lindgren (2016) lors de leur recherche réalisée dans huit cliniques de soins psychiatriques en Suède, dont l'objectif était d'explorer les processus vécus au quotidien par le personnel qui y travaille. Leurs observations montrent que lorsque le personnel rencontre des obstacles dans la mise en place du travail de collaboration, il devient alors incapable d'intervenir et s'éloigne des patients et des collègues. Dans la présente étude, une fois l'impasse thérapeutique identifiée, il devient possible, pour les professionnels, d'envisager des solutions. À partir de cycles d'actions et de réflexions, ils développent une meilleure pratique de collaboration et parviennent à dénouer leurs impasses thérapeutiques. Bouchard (2010) reconnaît qu'en contexte de travail d'équipe, les solutions aux impasses thérapeutiques émergent souvent des questionnements concernant la façon de travailler ensemble.

Enfin, l'étude appuie certaines recherches qui ont souligné l'importance des relations entre les individus comme un facteur pouvant influencer l'action collective dans le milieu de la santé (Tomizawa, Shigeta et Reeves, 2017). Toutefois, la recherche a également mis en évidence que les bonnes relations entre les individus ne sont pas suffisantes à la mise en place d'une pratique de collaboration lors des discussions sujettes aux impasses thérapeutiques. Le contexte de pratique est un facteur important dont il faut tenir compte pour offrir des moyens concrets (cadre 
théorique partagé, reconnaissance de zones spécifiques d'expertise, leadership orienté vers la collaboration, procédés d'animation en réunion) afin de soutenir les professionnels dans leur engagement dans la discussion et leur interdépendance. Ayant expérimenté une démarche où des professionnels sont invités à partager l'expérience de leurs interactions avec les patients et avec le personnel, Hern, Talen, Babiuch et Durazo-Arvizu (2009) confirment l'importance d'offrir aux professionnels des opportunités de développer leurs aptitudes à communiquer leur expérience et leurs aptitudes de résolution de problèmes afin qu'ils comprennent mieux ce qu'ils vivent dans leurs relations avec les clients et les collègues. Réfléchir ensemble et de façon structurée sur leur fonctionnement en équipe lors de discussions cliniques sujettes à une impasse thérapeutique rend possible l'ajustement de leurs pratiques et favorise le travail de collaboration (Reeves et Lewin, 2004).

\section{Forces et limites de la recherche}

Cette recherche-action participative menée avec une équipe en clinique de pédopsychiatrie et portant sur la collaboration interprofessionnelle comme modalité de résolution des impasses thérapeutiques présente un caractère original. En effet, s'il existe des études portant sur la collaboration interprofessionnelle dans les services de première ligne (Duclos, Lebeau et Guay, 2013; Morgan, Pullon et McKinlay, 2015) et entre les organisations (Fleury et Grenier, 2012; Fuller et al., 2011; Nadeau et al., 2012), aucune, à notre connaissance, ne s'est intéressée aux équipes cliniques en pédopsychiatrie en utilisant une modalité de recherche-action participative. Sur le plan méthodologique, la collaboration entre la chercheuse et les professionnels s'est avérée une stratégie gagnante qui a favorisé la production de connaissances pratiques et utiles permettant à l'équipe de professionnels de faire des changements dans leur fonctionnement en réunion d'équipe. Enfin, l'utilisation de l'incident critique, malgré son caractère confrontant, a donné accès à ce qui se vit réellement pendant ces réunions.

Les choix méthodologiques établissent aussi certaines limites dans cette étude. Tout d'abord, comme elle s'est penchée sur l'expérience d'une équipe de professionnels travaillant en clinique de pédopsychiatrie, ses résultats sont uniquement transférables à une population ayant des similitudes avec les participants à la recherche. La seconde limite concerne la participation des professionnels, qui n'a pas été constante tout au long du processus, pour les raisons évoquées précédemment. En effet, les professionnels participant aux réunions d'équipe et aux périodes de réflexion n'étaient pas nécessairement les mêmes d'un cycle à l'autre. Cette situation a eu une incidence sur la profondeur des réflexions: les nouveaux arrivants n'ayant pas nécessairement atteint le même niveau quant à leur capacité à porter attention aux actions et à réfléchir sur ces 
actions, et le processus de réflexion en équipe nécessitant la mise en place d'un certain climat de confiance entre participants, l'arrivée de nouveaux professionnels impliquait de travailler à reconstruire ce climat à chacun des cycles.

\section{CONCLUSION}

Cette recherche met en évidence la possibilité que le cheminement thérapeutique d'un patient soit influencé négativement lorsque les professionnels interdépendants d'une clinique de pédopsychiatrie rencontrent des difficultés à structurer leur action collective afin de répondre aux besoins de ce patient. Les obstacles à l'évolution positive du patient engendrent des coûts sociaux, psychologiques et financiers considérables (Cappelli et Léon, 2017). Ce constat rappelle donc la nécessité, pour les praticiens et gestionnaires, de mettre en place des moyens permettant l'implantation d'une réelle pratique de collaboration interprofessionnelle en clinique de pédopsychiatrie. La recherche souligne également l'impact positif que peut avoir la mise en place de lieux et d'espace-temps permettant aux professionnels de réfléchir ensemble sur leur pratique de collaboration, tant en ce qui a trait au déploiement d'une telle pratique que pour la résolution des impasses thérapeutiques qu'ils peuvent rencontrer.

Enfin, la collaboration interprofessionnelle comme modalité de résolution des impasses thérapeutiques en clinique de pédopsychiatrie mériterait d'être davantage explorée dans d'autres recherches. Si l'engagement des professionnels dans leur tâche à accomplir et la nécessité de tenir compte de leur interdépendance sont des facteurs favorables à la collaboration interprofessionnelle faisant généralement l'unanimité chez les chercheurs, il pourrait être intéressant d'examiner les contextes de pratique qui soutiennent le déploiement de ces facteurs ou encore d'examiner comment les mettre en place. II demeure également pertinent d'explorer davantage la collaboration interprofessionnelle comme modalité de résolution des impasses thérapeutiques rencontrées par les équipes. Ce type d'études gagnerait à s'échelonner sur une plus longue période de temps avec une plus grande stabilité des participants.

\section{RÉFÉRENCES}

Andvig, E., Syse, J. et Severinsson, E. (2014). Interprofessional collaboration in the mental health services in Norway. Nursing Research and Practice, Article 849375, Repéré à http://dx.doi.org/10.1155/2014/849375

Baum, F., MacDougall, C. et Smith, D. (2006, novembre). Participatory action research. Journal of Epidemiology \& Community Health, 854-857.

Beckett, P., Field, J., Molloy, L., Yu, N., Holmes, D. et Pile, E. (2013). Practice what you preach : Developing person-centred culture in inpatient mental health settings through strengths-based, transformational leadership. Issues in Mental Health Nursing, 34, 595601. doi:10.3109/01612840.2013.790524 
Bessette, M. (2010). Psychothérapie des troubles de la personnalité : Quand l'impasse n'est pas du côté du client. Santé mentale au Québec, 35(2), 87-116.

Bordeleau, L. et LeBlanc, J. (2017). La collaboration interprofessionnelle comme modalité pour résoudre les impasses thérapeutiques en pédopsychiatrie : une revue de littérature. Santé mentale au Québec, 42(2), 229-243.

Bouchard, S. (2010). Impasses et opportunités dans le traitement des personnes souffrant d'un trouble sévère de la personnalité limite. Santé mentale au Québec, 35(2), 61-85.

Cappelli, M. et Léon, S. L. (2017). Ouvrir la voie aux soins connectés : renforcer l'interface entre les soins primaires et les services de santé mentale communautaires pour enfants et adolescents. Repéré à http://www.excellenceforchildandyouth.ca/sites/default/files/ resource/politiques_soins_primaire.pdf

Careau, E., Vincent, C. et Swaine, B. R. (2011). Consensus group session of experts to describe interprofessional collaboration processes in team meetings. Journal of Interprofessional Care, 25, 299-301. doi: 10.3109/13561820.2011.566649

Cassell, C. et Johnson, P. (2006). Action research: Explaining the diversity. Human Relations, 59, 783-814.

Chou, F., Kwee, J., Buchanan, M. et Lees, R. (2016). Participatory critical incident technique: A participatory action research approach for counselling psychology. Canadian Journal of Counselling and Psychotherapy, 50(1), 51-74.

D'Amour, D. (1997). Structuration de la collaboration interprofessionnelle dans les services de santé de première ligne au Québec (Thèse de doctorat inédite). Université de Montréal, Montréal, QC.

D'Amour, D., Goulet, L., Labadie, J.-F., San Martín-Rodriguez, L. et Pineault, R. (2008). A model and typology of collaboration between professionals in healthcare organizations. BMC Health Services Research, 8, 188. doi: 10.1186/1472-6963-8-188

Duclos, C., Lebeau, A. et Guay, M. (2013). Étude des modes d'organisation des équipes de première ligne en santé mentale jeunesse en Montérégie. Santé mentale au Québec, 38(1), 61-80.

Flanagan, J. C. (1954). The critical incident technique. Psychological Bulletin, 51(4), 327-358.

Fleury, M.-J. et Grenier, G. (2012). État de situation sur la santé mentale au Québec et réponse du système de santé et de services sociaux. Repéré à http://www.csbe.gouv.qc.ca

Fradet, L. (2013). Quelle approche de synthèse des connaissances adopter pour faire un état des lieux de la recherche-action participative en santé et services sociaux au Québec francophone? Nouvelles pratiques sociales, 25(2), 219-230.

Fuller, J. D., Perkins, D., Parker, S., Holdsworth, L., Kelly, B., Roberts, ... Fragar, L. (2011). Building effective service linkages in primary mental health care : A narrative review part 2. BMC Health Services Research, 11, 66. doi: 10.1186/1472-6963-11-66.

Gardner, A. K., Scott, D. J. et AbdelFattah, K R. (2016). Do great teams think alike? An examination of team mental models and their impact on team performance. Surgery, 161(5), 1203-1208. doi: 10.1016/j.surg.2016.11.010

Hern, T., Talen, M., Babiuch, C. et Durazo-Arvizu, R. (2009, septembre). Patient care management teams: Improving continuity, office efficiency, and teamwork in a residency clinic. Journal of Graduate Medical Education, 67-72. doi: 10.4300/01.01.0011

Hesjedal, E., Hetland, H. et Iversen, A. C. (2015). Interprofessional collaboration: Selfreported successful collaboration by teachers and social workers in multidisciplinary teams. Child and Family Social Work, 20, 437-445. doi:10.1111/cfs.12093

Jagosh, J., Macaulay, A. C., Pluye, P., Salsberg, J., Bush, P. L., Henderson, J., ... Greenhalgh, T. (2012). Uncovering the benefits of participatory research : Implications of a realist review for health research and practice. The Milbank Quarterly, 90(2), 311-346.

Lesinskiene, S., Senina, J. et Ranceva, N. (2007). Use of the HoNOSCA scale in the teamwork of inpatient child psychiatry unit. Journal of Psychiatric and Mental Health Nursing, 14, 727-733.

Lincoln, Y. S. et Guba, E. G. (1985). Naturalistic inquiry. Beverly Hills, CA : Sage.

MacDonald, C. (2012). Understanding participatory action research : A qualitative research methodology option. Canadian Journal of Action Research, 13(2), 34-50. 
Manion, I. G. (2010). Provoking evolution in child and youth mental health in Canada. Canadian Psychology, 51(1), 50-57.

Marks, M. A., Mathieu, J. E. et Zaccaro, S. J. (2001). A temporally based framework and taxonomy of team processes. Academy of Management Review, 26(3), 356-376.

McComb, S. et Simpson, V. (2014). The concept of shared mental models in healthcare collaboration. Journal of Advanced Nursing, 70(7), 1479-1488. doi: 10.1111/jan.12307

Mclnnes, S., Peters, K., Bonney, A. et Halcomb, E. (2015). An integrative review of facilitators and barriers influencing collaboration and teamwork between general practitioners and nurses working in general practice. Journal of Advanced Nursing, 71(9), 1973-1985.

Meyer, J. (2000). Using qualitative methods in health related action research. British Medical Journal, 320(7228), 178-181.

Ministère de la Santé et des Services sociaux (MSSS). (2005). Plan d'action en santé mentale 2005-2010 : La force des liens. Repéré à http://publications.msss.gouv.qc.ca/

Ministère de la Santé et des Services sociaux (MSSS). (2010). Évaluation de l'implantation des réseaux locaux de services de santé et des services sociaux. Repéré à http://publications.msss.gouv.qc.ca/

Ministère de la Santé et des Services sociaux (MSSS). (2015). Plan d'action en santé mentale 2015-2020 2020: Faire ensemble et autrement. Repéré à http://publications.msss.gouv.qc.ca/

Molin, J., Graneheim, U. H., Ringner, A. et Lindgren, B.-M. (2016). From ideals to resignation - interprofessional teams perspectives on everyday life processes in psychiatric inpatient care. Journal of Psychiatric and Mental Health Nursing, 23, 595-604. doi: 10.1111/jpm.12349

Morgan, S., Pullon, S. et McKinlay, E. (2015). Observation of interprofessional collaborative practice in primary care teams: An integrative literature review. International Journal of Nursing Studies 52, 1217-1230.

Murray, S., Silver, I., Patel, D., Dupuis, M., Hayes, S. M. et Davis, D. (2008). Community group practices in Canada: Are they ready to reform their practice? Journal of Continuing Education in the Health Professions, 28(2), 73-78.

Nadeau, L., Jaimes, A., Rousseau, C., Papazian-Zohrabian, G., Germain, K., Broadhurst, ... Measham, T. (2012). Partnership at the forefront of change: Documenting the transformation of child and youth mental health services in Quebec. Journal of the Canadian Academy of Child \& Adolescent Psychiatry, 21(2), 91-97.

Organisation mondiale de la santé (OMS). (Août 2014). 10 faits sur la santé mentale. Repéré à http://www.who.int/features/factfiles/mental_health/fr/

Paillé, P. et Muchielli, A. (2008). L'analyse qualitative en sciences humaines et sociales. Paris, France : Armand Colin.

Rasic, D. (2010). Countertransference in child and adolescent psychiatry-a forgotten concept? Journal of the Canadian Academy of Child \& Adolescent Psychiatry, 19 (4), 249-254.

Reeves, S. et Lewin, S. (2004). Interprofessional collaboration in the hospital: Strategies and meanings. Journal of Health Services Research \& Policy, 9(4), 218-225.

Rober, P. (1999). Le dialogue intérieur du thérapeute dans la pratique de la thérapie familiale : quelques idées sur le Moi du thérapeute, l'impasse thérapeutique et le processus de réflexion. Thérapie Familiale, 2, 131- 154.

Rousseau, C., Nadeau, L., Pontbriand, A., Johnson-Lafleur, J., Measham, T. et Broadhurst, J. (2014). La santé mentale jeunesse: un domaine à la croisée des chemins. Santé mentale au Québec, 39(1), 101-118.

Sicotte, C., D'Amour, D. et Moreault, M.-P. (2002). Interdisciplinary collaboration within Quebec community health care centres. Social Science \& Medicine, 55, 991-1003.

Sidani, S. et Fox, M. (2014). Patient-centered care : Clarification of its specific elements to facilitate interprofessional care. Journal of Interprofessional Care, 28 (2), 134-41.

Sims, S., Hewitt, G. et Harris, R. (2015). Evidence of collaboration, pooling of resources, learning and role blurring in interprofessional healthcare teams: A realist synthesis. Journal of interprofessional care, 29(1), 20-25. doi: 10.3109/13561820.2014.939745

St-Arnaud, Y. (2008). Les petits groupes, participation et animation (3e éd.). Montréal, QC : Gaëtan Morin. 
Suter, E., Arndt, J., Arthur, N., Parboosingh, J., Taylor, E. et Deutschlander, S. (2009). Role understanding and effective communication as core competencies for collaborative practice. Journal of Interprofessional Care, 23(1), 41-51.

Toegel, G. et Barsoux, J.-L. (2016). How to preempt team conflict. Harvard Business Review. 94(6), 78-83.

Tomizawa, R., Shigeta, M. et Reeves, S. (2017). Framework development for the assessment of interprofessional teamwork in mental health settings. Journal of Interprofessional Care, 31(1), 43-50. doi: 10.1080/13561820.2016.1233098

Walker, J. (2015) Using Critical Incidents to Understand ESL Student Satisfaction. TESL Canada Journal/Revue TESL du Canada. 32(2), 95-111.

Washington, K., Guo, Y., Albright, D. L., Lewis, A., Oliver, D. P. et Demiris, G. (2017). Team functioning in hospice interprofessional meetings : An exploratory study of providers' perspectives. Journal of Interprofessional Care, 31(4), 455-462. Repéré à http://dx.doi.org/10.1080/13561820.2017.1305950

Zwarenstein, M. et Reeves, S. (2006). Knowledge translation and interprofessional collaboration: where the rubber of evidence-based care hits the road of teamwork. Journal of Continuing Education in the Health Professions, 26, 46-54.

\section{RÉSUMÉ}

Cet article présente les fondements, la méthode et les résultats d'une recherche-action participative visant à comprendre comment des professionnels travaillant dans une clinique de pédopsychiatrie construisent leur pratique de collaboration interprofessionnelle, le contexte dans lequel se construit cette pratique et comment ils peuvent améliorer la résolution des impasses thérapeutiques en réfléchissant sur cette pratique. Un traitement qualitatif, à partir du procédé d'analyse thématique de données recueillies au cours de périodes de réflexion en équipe et d'une entrevue de groupe, a permis d'identifier sept déterminants du processus de collaboration interprofessionnelle regroupés selon trois thèmes : 1) l'engagement, 2) les interactions et 3) le contexte de pratique. Six déterminants favorisent le travail de collaboration interprofessionnelle, alors qu'un y fait obstacle. La mise en évidence des déterminants au cours du déroulement de la recherche a un impact positif sur le processus de collaboration interprofessionnelle et sur la résolution des impasses thérapeutiques. Les forces et les limites de la recherche, ses retombées pour la pratique et des pistes pour de futures recherches sont présentées.

\section{MOTS CLÉS}

collaboration interprofessionnelle, impasse thérapeutique, pédopsychiatrie

\section{ABSTRACT}

This article presents the theoretical background, methodology and results of a participatory action research aimed at understanding how professionals working in a child psychiatry clinic build their interprofessional collaboration practice, the context in which this practice is built, and how these professionals can, by reflecting on their practice, improve the resolution of therapeutic impasses. Through a qualitative analysis, based on a thematic analysis methodology of data collected during team reflection periods and a group interview, we identified seven determinants of the interprofessional collaboration process grouped into three themes: 1) commitment, 2) interactions and 3) the practice context. Six determinants favour collaborative interprofessional work while one determinant acts as an obstacle. Evidencing these determinants during the course of research had a positive impact on the interprofessional collaboration process and the therapeutic impasses resolution. The research strengths and limitations, consequences for practice and avenues for future research are presented. 
Collaboration interprofessionnelle et pédopsychiatrie

\section{KEYWORDS}

interprofessional collaboration, therapeutic impasse, child psychiatry 